\title{
Study on the Coupling Mechanism of Spatial Form and Mesoscale Wind Environment in Coastal Cities Based on Multivariate Analysis: Progress
}

\author{
Xiaowan $\mathrm{Han}^{1, \mathrm{a}}$, $\mathrm{Ke} \mathrm{He}^{2, \mathrm{~b}}$, Yanting $\mathrm{He}^{3, \mathrm{c}}$ and Chunxi Zhao ${ }^{4, \mathrm{~d}}$ \\ ${ }^{1}$ Huaqiao University, No.668, Jimei Avenue, Jimei District, Xiamen, Fujian Province \\ ${ }^{2}$ North Gate Construction Building, No. 3688, Nanhai Avenue, Nanshan District, Shenzhen, \\ Guangdong Province \\ ${ }^{3}$ School of Civil Engineering, Beifang University of Nationalities, No. 204, Wenchang North Street, \\ Xixia District, Yinchuan, Ningxia Hui Autonomous Region \\ ${ }^{4}$ North China University of Technology, No. 5, Jinyuanzhuang Road, Shijingshan District, Beijing \\ a2512034217@qq.com, bhkqq5@qq.com, c15594735@qq.com, d43062282@qq.com
}

\begin{abstract}
Key words: Mesoscale Wind Environment, Urban Spatial Form, Multivariate Analysis; Research Method
\end{abstract}

Abstract. Chinese and foreign academic circles have made certain progress in the study of mesoscale wind environment and urban spatial form. However, due to the different research background and approach, the academic achievements made in this field vary to some extent in China and abroad. In China, the academic community has upgraded the research of urban spatial form from qualitative research to quantitative analysis. At present, domestic urban wind environment research is focused on building scale and small scale urban wind environment research.

\section{Introduction}

The industrial structure of China's coastal cities is generally industrial-based, and the government's study of environmental pollution lacks depth and width. Therefore, although coastal areas themselves are conducive to the dilution, diffusion and removal of air pollutants, the haze weather is still on the rise. Furthermore, with the rapid development of tourism, the pollutant emissions is significantly larger than other cities, which has a great impact on the urban environment. Urban wind environment, as an important factor affecting the urban environment and unarguably the coastal city environment, plays a significant role in the spread of air pollutants, heat island effect mitigation, open space thermal comfort perception and high-rise building wind safety. A good urban wind environment, especially the wind environment at pavement level, can provide a more healthy and comfortable outdoor activity platform for urban residents. Urban wind environment is mainly affected by the urban spatial form. A reasonable urban design can effectively promote the city ventilation to improve urban air quality. However, in order to come out with the urban design method for urban spatial form based on the urban wind environment, we have to first of all study the coupling mechanism of urban spatial form and urban wind environment. For decades, scholars at home and abroad have done a lot of research in this field, and achieved great results. This paper analyzes the research papers of mesoscale wind environment and urban spatial form in order to provide general information about the current state of mesoscale wind environment and urban spatial form in China, and find out the shortcomings to put forward suggestions for the research in the future.

\section{Research methods}

In order to understand the overall research level and recent research direction of urban wind environment and urban spatial form in our country, we have analyzed the number of papers published over the past two decades in this field in our country. In order to avoid overlapped statistics and ensure the accuracy of statistical results, we only chose the knowledge service platform of Chininfo as the data source in the literature search. In the process of data statistics, we 
searched the literatures on the conditions that set "urban wind environment" and/or "urban spatial form" as the subject, containing "quantitative" or "multivariable" in the abstract for accurate match, and especially set the subject domain as all albums for all the related literatures in the past considering the role of the urban wind environment in various fields. After obtaining the relevant literatures, we judged if they were related to the subject of research by the topics and subtracts of the papers, and then started multi-perspective analysis of the literature samples after ruling out the irrelevant literatures.

\section{The current situation and development of the studies abroad}

Research on the relationship between urban wind environment and urban spatial form. The urban wind environment analysis technology in western countries has been developing on the basis of urban climate research: Since the 1960s, the wind tunnel experiment has been introduced into the study of urban wind environment to realize the accurate simulation of the airflow around the buildings. Since the 1990s, the research on the relationship between the urban wind environment and urban spatial form based on computer numerical simulation has begun, mostly focusing on micro-scale or local wind environment. For example, Air Flow around Isolated Gable-roof Buildings with Different Roof Pitches: Wind Tunnel Experiments and CFD Simulations (Tominaga et.al., 2014[1]) which studies the air flow around the globle-roof buildings through the wind tunnel test and computation of fluid dynamics (CFD), Numerical Simulation of Three - dimensional Steady - wind field in High - rise Buildings (Yang Wei, Gu Ming, 2003 [2]) which compares CFD simulation with wind tunnel experiment; "Beijing business center wind environment wind tunnel experimental study" (Wang Baomin et al., 2004 [3] which studies of the pedestrian wind environment in Beijing Business Center by wind tunnel erosion technology; Study on the Simulation and Optimization Design of Human Wind Environment in High-rise Buildings Based on CFD Numerical Simulation Method (Yang Yi, Jin Xinyang, Yang Liguo, etc., 2011 [4]) which is a study on the outdoor wind environment of the research buildings of the China Academy of Building Research by the CFD numerical simulation method. Although China is late to apply CFD, it is gradually growing up. As indicated by the statistics of wind environment study in China in previous years, CFD as a powerful numerical simulation tool, has gradually replaced the traditional research methods, and saw a sharp increase in 2012. (Fig. 1)

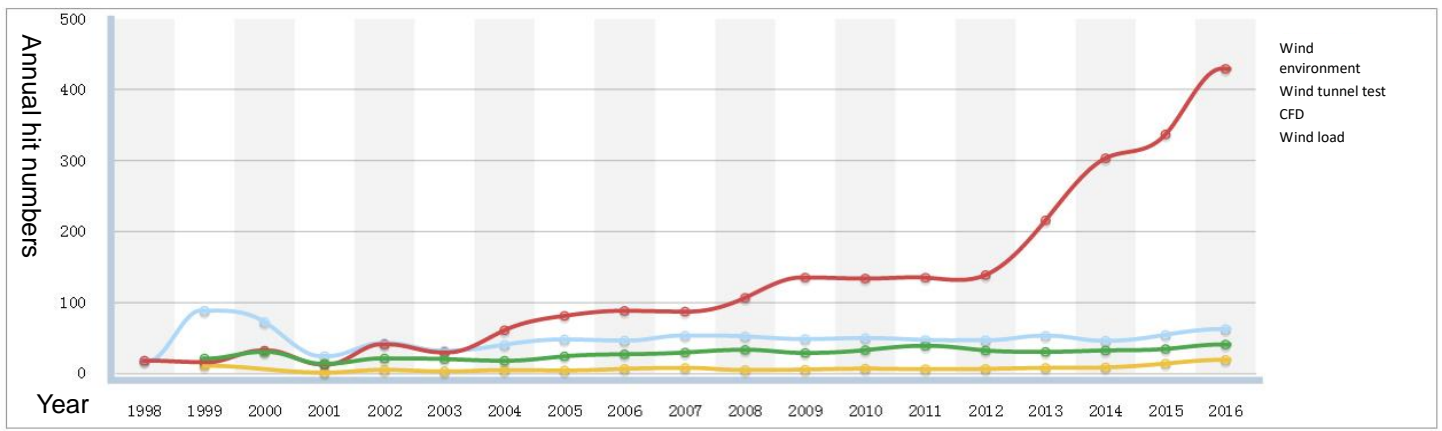

Fig. 1 Wind environment research trend in China (source: Chinainfo)

With the development of urbanization, the interference of urban construction on urban airflow is increasing, which leads to further discussion of the urban wind environment, and links to urban wind environment through the criteria related to urban morphology. For example, Impact of Urban Spaces and Building Height on Airflow Distribution: Wind Tunnel Testing of an Urban Setting Prototype in Abu-Dhabi City (Khaled A, 2001[5]) is an analysis of air velocity and outdoor thermal comfort of the city of Abu Dhabi through the study of urban building height, space width and other parameters and The Study on Application of wind environment simulation in the optimization of urban spatial form - A Case Study of the Experimental Ecological Community of Chenjia Town, Chongming, Shanghai (Liu Chao, et.al. 2013 [6]), which is an analysis of the inefficiency of urban spatial form in providing the comfort of wind environment, and in turn puts forward the strategy of 
optimizing the form. The study of mesoscale wind environment is also involved in these reports, which emphasize the influence of mesoscale top-surface form of urban local areas and blocks on urban local wind environment. For example, The Study of the Characteristics of the Near-earth Wind Field in Guangzhou (Feng Xianhui, Wei Qingquan, 2011 [7]), which is a study of how to use of urban layout to improve the city's near-earth wind field. In addition to the technical analysis of urban wind environment, these studies also show that there is a close and complex coupling relationship between urban spatial form and urban wind environment. This provides an important reference value and guidance for the study in the future. We will use the well-equipped facilities of Haixi Institute of Science and Technology of Xiamen University of Science and Technology to further discusses the coupling mechanism between urban spatial form and urban wind environment on the basis of the existing national special research program A Study on the Near-earth Wind Environment of Xiamen Digital City Based on Multivariate Analysis (He Ke, SAKAI Takeru, 2016 -2018).

Quantitative study of urban spatial form gradually replaced by a quantitative one. Before the 1950 s, the study of western urban spatial morphology was mostly one-way static qualitative research. Then, the research method of urban spatial form shifted from simple qualitative description to precise quantitative analysis. A group of scholars have established a variety of mathematical models. For example, Microsimulation of Urban Development and Location Choices: Design and Implementation of UrbanSim (P. Waddell et al., 2003 [11]) is an urban simulation model to coordinate traffic and land use planning. Since GIS technology was introduced into urban planning in the 1980s, the widespread use of urban spatial analysis in recent years has spawned the emergence of quantitative urban form analysis methods, from which an approach to quantitative analysis of single urban spatial morphological elements gradually emerged. For example, Towards a Multimodal Space Syntax Analysis: A case study of the London Street and Underground Network ( S Law, AJ Chiaradia, 2012[12]) is the application of spatial syntax in the analysis of street space. In recent years, quantitative analysis of two or more urban spatial morphological elements has also been developed. For example, The Theory of the Natural Urban Transformation Process: The Relationship Between Street Network Configuration, Density and Degree of Function Mixture of Built Environments (Ye Y, Van Nes A. 2014[13]) is a quantitative analysis of the various elements in a series of Dutch towns. China's research on the development of urban spatial form is slower than that of the West. After the 1980s, the study of urban morphology began to receive attention. Since the 1990s, urban planning and construction of various fields have begun to quantify study of the urban spatial form. For example, The Quantitative Study of Urban CBD Spatial Form - the Second Part of quantitative Research on CBD Development in China (Yang Junjie, Wu Mingwei, 2006 [14]) is a quantitative study of CBD spatial form in terms of CBD location, layout and vitality of CBD. The Analysis of Xiamen Urban Spatial Form Based on Spatial Syntaxv Analysis (He Zizhang et al., 2007 [15]) is a syntax analysis of Xiamen urban spatial form and a summary of Xiamen urban form development. The Quantitative Analysis of Urban Street Network Spatial Form of Quantitative Analysis of Complex Spatial Characteristics of Urban Streets (Yuan Si-nan, 2011 [16]) is a quantitative analysis of the characteristics of the complex network spatial form by computer-based technology. The Construction of the Method for the Analysis of the Spatial Form Evolution of the Open Port Cities based on GIS and VR technology (Hou Xin et al., 2013 [17]) is a study framework for construction of the spatial form of modern open port cities of China by means of 3D digital model and GIS technology.

In recent years, China's microclimate study of urban spatial morphology has been rolled out gradually. For example, The Study on the Correlation between the Urban Spatial Morphology and Urban Micro-environment (Ding Wowo, Hu Youpei, Dou Pingping, 2012 [18]) is an approach to quantify the sky openness, texture roughness and other urban morphological factors to determine the correlation factors between urban spatial form and urban micro-environment, indicating that the academic community has recognized that the quantitative study of urban spatial form in the context of the urban wind environment research is of great significance. It has provided valuable information basis for this subject to establish specific evaluation indicators for quantitative analysis 
of the specific evaluation index of this subject, and also reflected the urgent demand of our country's application of the quantification of urban spatial form. We have also made some positive explorations in this field. For example, The Digital Analysis of the Spatial Form of the Ancient City Walls in the Southeastern China Based on Multivariate Analysis (He Ke, Wang Chengkang et al. 2015-1017) is an approach to study urban spatial form based on quantitative study on the spatial compactness of urban space based on multivariate analysis. The Quantitative Study of the compactness of Urban Spatial Form Based on the Mesoscopic and Microscopic Scale Evaluation (Jin Jun, He Ke et al., 2014-2016) is a quantitative study of the urban spatial form of the mesoscopic and microscopic scales and The Theory to Analyze Traditional Residential Blocks and Study of its Conservation and Upgrade from the Perspective of Property Ownership (Jiang Hong, He Ke et al., 2014-2016) is a study of the urban spatial form of the traditional residential areas. Based on the above studies, this study will continue to improve multivariable analysis technology, define the parametric system of each element of urban spatial form and accurately describe the general characteristics of the spatial form in coastal cities.

The Application of Interdisciplinary Multivariate Theory in its Early Stage. Multivariate analysis is now in a stage of wide application since its theoretic development in the 1930s and computer-based application in the 1970s. It is not easy to directly apply the multivariate analysis theory of the West to the study of urban spatial form in China because the development background of Western developed countries is different from that of China. Japan has conducted more in-depth research on multivariate analysis of urban spatial form applications and is committed to simplifying the underlying theoretical model. For example, professors, such as IKARUGA Shinji, SAKAI Takeru, conducted quantitative analysis of landscape elements through the study of traditional landscape painting. Professors, such as KOIZUMI Koji, divided and quantified the elements of the street space and discussed the relationship between the elements of the street space and the formation of the street atmosphere. Professors, such as KIM Joonho, focused on the elements of the rest area of the storefront, and described the effect of the rest area on the user's behavior through investigating and analyzing the use of the rest area. China's multivariate analysis is widely applied in many industries, and is in a nascent stage of exploring the application of the urban spatial form analysis. For example, A Study on the Impact of Traffic in Beijing on Urban Spatial Form (Hou Min, Zhu Rongfu, 2007 [24]) reveals how different traffic is affecting the urban spatial form by introducing multivariate logistic regression model to calculate the influence coefficient of the traffic factors on the spatial form of urban space. Simulation and Prediction of Urban Spatial Expansion (Yan Mei, 2012 [25]) establishes a logical regression model of urban spatial expansion simulation to simulate and predict the urban spatial expansion.

We will learn from the experience of Japanese who are close to us in the cultural background and the study progress made by us through a partnership with Professor SAKAI Takeru of Kyushu University. For example, Digital Analysis of the Spatial Form of Ancient City Walls in Southeast China Based on Multivariate Analysis (He Ke, Wang Chengkang et al., 2015-2017) is an approach to the study of urban spatial form based on multivariate analysis as described above. The Inspiration of Arcade Street in Japan to the Transformation of Traditional Chinese Districts (He Ke, Wang Chengkang et al., 2011) is the initial attempt to quantify the spatial form of historical blocks. Re-evaluation of Loop-shaped Urban Structure of Compact City as Infrastructure of Mature Society (Wang Chengkang, He Ke et al., 2009-2011) is a typological analysis of Nanjing wall space using the clustering methodology of multivariate analysis, and gives advice for its protection and utilization. We will further apply the multivariable theory to the study of the coupling mechanism of urban spatial form and mesoscale wind environment, and clarifies and simplifies the digital process of "screening variables - quantitative indicators - clear mechanism - determining model" to establish and optimize the guidance system for multivariable spatial morphology of China's coastal urban wind environment.

\section{Conclusions}

In view of the general situation, Chinese and foreign academic circles have a certain basis for the 
study of mesoscale wind environment and urban spatial form, but because of the different research background and research approach, the academic results show a certain difference. According to the search of the authoritative search platform, there are nearly 3,000 papers published by the domestic scholars about the mesoscale urban wind environment and the coastal city space form, of which the study of mesoscale wind environment is characterized by multi-disciplinary research (Fig. 2). The study of coastal space form is based on a qualitative study (Fig. 3), and quantitative research is increasing year by year. In general, further quantitative research on the coupling of the mesoscale wind environment and the urban spatial form in China should be improved and upgraded, and it has the research foundation under the background of the current age.
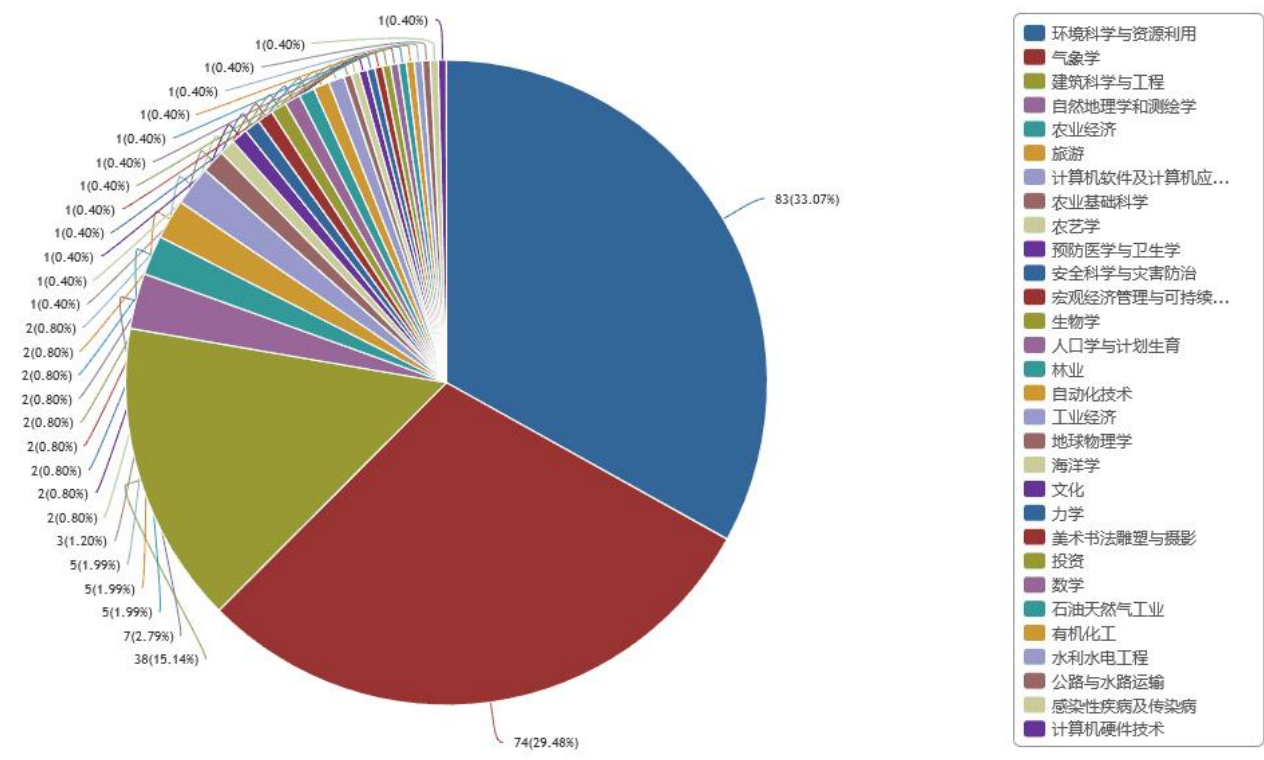

Fig. 2 Distribution of mesoscale urban wind environment subject (data source: Chinainfo)
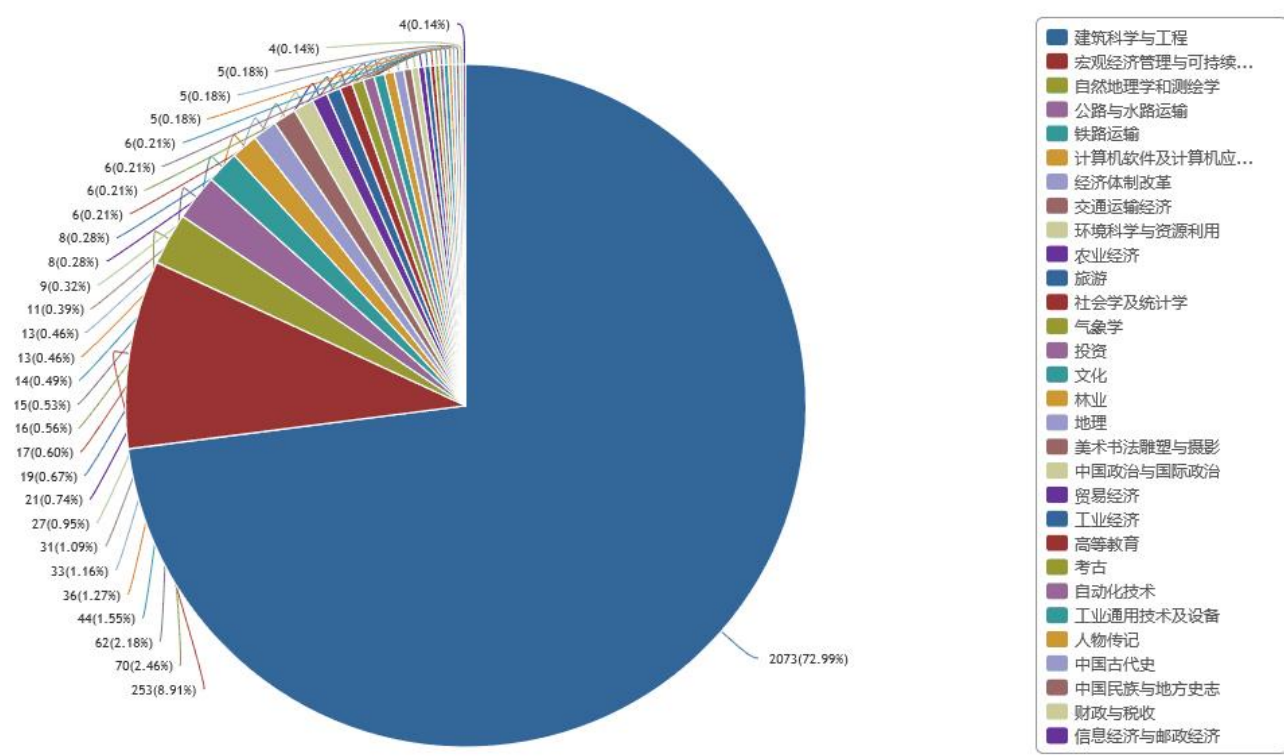

Fig. 3 Distribution of coastal city spatial morphology (data source: Chinainfo) 


\section{Acknowledgements}

This work was financially supported by the National Natural Science Foundation (51408118) and the State Bureau of Foreign Experts in High-end Projects (GDT20163600002) of China.

\section{References}

[1] Tominaga Y, Akabayashi S I, Kitahara T, et al. Air flow around isolated gable-roof buildings with different roof pitches: Wind tunnel experiments and CFD simulations[J]. Building \& Environment, 2014, 84:204-213.

[2] Yang Wei, Gu Ming. Numerical simulation of three-dimensional steady wind field in high-rise buildings [J]. Journal of Tongji University Natural Science Edition, 2003, 31 (6): 647-651.

[3] Wang Baomin, Liu Huizhi, Sang Jianguo, et al.Experimental study on wind tunnel in Beijing business center [J]. Climate and Environment Research, 2004, 9 (4): 631-640.

[4] Yang Yi, Jin Xinyang, Yang Li-guo, et al.Study on pedestrian wind environment simulation and optimization design of high-rise buildings [J]. Building Science, 2011, 27 (1): 4-8.

[5] Khaled A. Al-Sallal, Mohsen M. AboulNaga, Abdullatif M. Alteraifi. Impact of Urban Spaces and Building Height on Airflow Distribution: Wind Tunnel Testing of an Urban Setting Prototype in Abu-Dhabi City[J]. Architectural Science Review, 2001, 44(3):227-232.

[6] Liu Chao, Chen Weizhen, Xu Peng, et al.Application of Wind Environment Simulation in Urban Spatial Form Optimization - A Case Study of Experimental Ecological Community in Chenjia Town, Chongming, Shanghai [C] 2013 China Urban Planning Annual Conference . 2013.

[7] Feng Xianhui, Wei Qingquan, A Study of the Characteristics of the Near-ground Wind Field in Guangzhou [J]. Journal of Eco-Environmental Sciences, 2011, 20 (10): 1558-1561.

[8] Tian Yuanyuan, Xu Weiquan. Experimental study on human body thermal reaction in hot and humid environment [J]. HVAC, 2003, (04): 27-30.

[9] Yang Wei, Gu Ming, Numerical Simulation of Three-Dimensional Steady Wind Field[J], Journal of Tongji University (Natural Science Edition), 2003, (06): 647-651.

[10] Zhou Li, Xi Guang; Numerical analysis of wind farm in high-rise buildings [J]. Journal of Xi'an Jiaotong University, 2001, (05): 471-474.

[11] Waddell P, Borning A, Noth M, et al. Microsimulation of Urban Development and Location Choices: Design and Implementation of UrbanSim[J]. Networks and Spatial Economics, 2003, 3(1):43-67.

[12]Law S, Chiaradia A J, Schwander A. Towards a multimodal space syntax analysis: A case study of the London street and underground network[J]. Dissertations \& Theses - Gradworks, 2012.

[13] Van Nes A, Ye Y. The theory of the natural urban transformation process: The relationship between street network configuration, density and degree of function mixture of built environments[J]. Aesop, 2014.

[14] Yang Jun-yan, Wu Mingwei. Quantitative Study on Spatial Form of Urban CBD: A Quantitative Stdy of CBD Development in China (Part 2); [J] .Journal of Urban Planning, 2006, 30 (2): $18-25$.

[15] HE Zizhang, Qiu Guochao, Yang Zhe. Study on the Development of Xiamen City's Form Based on Spatial Syntax Analysis [J]. Central China Architecture, 2007, 25 (3): 106-108.

[16] Yuan Sinan; Quantitative Analysis of Urban Street Network Space Form [D]; Tianjin University, 2011.

[17] Hou Xin, Wang Xiao-ping, Wang Xuan. Methodology of Evolutionary Analysis of Open-port City Space Form Evolution Based on GIS and VR Technology [J]; Journal of Tianjin University (Social Science Edition), 2013, 15 (3): 270-276.

[18] Ding Wowo, Hu Youpei, Dou Ping-ping. Study on the Correlation between Urban Morphology and Urban Microclimate [J] .Journal of Architecture, 2012 (7): 16-21.

[19] Wang Jincan. Spatial interpretation of Shenzhen Dongmen commercial pedestrian street based on spatial syntax [D]. Harbin Institute of Technology, 2012.

[20] Wang Jianfeng, Ai Jixi. Quantitative analysis of urban spatial form [J]. Chongqing Architecture, 2004, (S1): 7-10. 
[21] Wang Jianguo, Cai Kaizhen.Application of digital technology in modern urban design [J]. South Architecture, 2008, (02): 28-32.

[22] Shao Runqing, Duan Jin. Exploration of Penglai three-dimensional space shape and landscape characteristics of planning based on the openness [J] .Planner, 2011, (12): 44-50.

[23] Japanese Society of Architecture. Japan Institute of Architecture, Department of Environmental Studies [M]. Japan Institute of Architecture, 2003.

[24] Hou Min, Zhu Rongfu.Study on the Influence of Traffic on Urban Spatial Form in Beijing [J]. Bulletin of Surveying and Mapping, 2007 (12): 59-61.

[25] Yan Mei.Study on the prediction of urban spatial expansion [D]. Southwest University, 2012. 